Editorial

\title{
Insights into traditional Chinese medicine-microbe interaction using traditional Chinese medical microecology
}

\section{Editorial}

Traditional Chinese medical microecology (TCMM), a new discipline, is proposed by Prof. Lu-Qi Huang and Dr. Chang-JiangSheng Lai at June 2018. ${ }^{1}$ This new discipline is focused on the interaction between the microbe with the cultivation, production, quality, efficacy, safety, and origin of traditional Chinese medicine (TCM), as well as the TCM and the human microecology. In other words, the human micro-ecosystem, TCM micro-ecosystem, and therapeutic application are the major theoretical structure of TCMM. It is a new insight into development of the microbe for drug discovery and the therapeutic application of TCM.

Currently, there are three main research tasks in TCMM. Firstly, the microbe database of herbal and fungal TCM should be developed, including the soil or atmospheric microbe, plant endophyte, and microecological structure environmental factors. Secondly, the relationship between microecology and quality utility of TCM should be discoursed. The microecology and the microbial functional genes involved in active/ toxic compound metabolism, ${ }^{2-5}$ growth regulation, ${ }^{6,7}$ and pest/disease resistance play an important role in drug discovery, cultivation, and medicinal plant physiology and pathology, respectively. ${ }^{8,9}$ Thirdly, the interaction of TCM and human microecology should be viewed for a wide knowledge that the regulation effect of TCM on human intestinal flora, the disposal mechanism of human microorganism on $\mathrm{TCM},{ }^{10,11}$ and the human microecology reconstruction using TCM microbe therapy. Human intestinal flora has become a new target for further understanding human physiology and pathology, opening up a new way for the treatment of clinical diseases and greatly promoting the development of preventive and health care medicine. ${ }^{12,13}$

In TCMM studies, many difficulties should be solved in our further works. The growth conditions of many strains are difficult to simulate artificially and cannot be purified. Moreover, the endophytes of medicinal plants often grow poorly and metabolize badly when they leave special environment of their host. Modern molecular biology techniques, such as high-throughput sequencing, ${ }^{14}$ have initially shown great diversity in the microecological flora of medicinal plants. These microorganisms can promote and regulate the growth of host plants or produce special secondary metabolites by mediating or assisting host plants. ${ }^{15}$ Some of these chemical components promote increase of the resistance for the medical plants, ${ }^{16}$ and some others are used as effective medical components for the prevention and treatment of human diseases. ${ }^{17,18}$ Notably, certain microbes may enter the human gut through oral administration, which may affect the intestinal flora. In total, the TCMM gives us a new way to discourse and modernize the TCM for human health.

\section{Acknowledgements}

This work was financially supported by the National Natural Science Foundation of China $(81603293,81673553)$ and Key project
Volume II Issue 4 - 2018

\author{
Chang-Jiang-Sheng Lai,' Dong-Mei He, ,'2 Lu- \\ Qi Huang' \\ 'National Resource Center for Chinese Materia Medica, China \\ ${ }^{2}$ Chengdu University of TCM, China
}

Correspondence: Chang-Jiang-Sheng Lai, National Resource Center for Chinese Materia Medica, China Academy of Chinese Medical Sciences, State Key Laboratory Breeding Base of Dao-di Herbs, China, Email laichangjiang44@।26.com

Received:June 10,2018 | Published: July II, 2018

at central government level: The ability establishment of sustainable use for valuable Chinese medicine resources (2060302).

\section{Conflict of interest}

The author declares no conflict of interest.

\section{References}

1. He DM, Lai CJS, Yan ZY. Research and prospect of traditional Chinese medical microecology. China J Chin Mater Med. 2018.

2. Gao H, Li G, Lou HX. Structural diversity and biological activities of novel secondary metabolites from endophytes. Molecules. 2018;23(3):646.

3. Hyde KD, Soytong K. The fungal endophyte dilemma. Fungal Divers. 2008;33:163-173.

4. Tejesvi MV, Segura DR, Schnorr KM, et a1. An antimicrobial peptide from endophytic Fusarium tricinctum of Rhododendron tomentosum Harmaja. Fungal Diversity. 2013;60(1):153-159.

5. Najjar A, Abdullah N, Saad WZ, et al. Detoxification of toxic phorbol esters from Malaysian Jatropha curcas Linn. kernel by Trichoderma spp. and endophytic fungi. Int J Mol Sci. 2014;15(2):2274-2288.

6. Sneha Ogale, Karan Singh Yadav, Shrutika Navale. Screening of endophytic bacteria from the pharmacologically important medicinal plant Gloriosa superba for their multiple plant growth promoting Properties. The Pharma Innovation Journal. 2018;7(1):208-214.

7. Pavlova AS, Leontieva MR, Smirnova TA, et al. Colonization strategy of the endophytic plant growth promoting strains of Pseudomonas fluorescens and Klebsiella oxytocaon the seeds, seedlings and roots of the epiphytic orchid, Dendrobium nobile Lindl. J Appl Microbiol. 2017;123(1):217-232.

8. Meena N, Saharan BS. Effective Biocontrol of leaf rot disease on Aloevera plant by PGPR in green house experiment. Bull Env Pharmacol. Life Sci. 2018;7(1):24-28.

9. Mishra A, Singh SP, Mahfooz S, et al. Endophyte-mediated modulation of defense-responsive genes and systemic resistance in Withania somnifera (L.) Dunal under Alternaria alternata stress. Appl Environ Microb. 2018;84(8):e02845-02917. 
10. Chan CL, Gan RY, Shah NP, et al. Polyphenols from selected dietary spices and medicinal herbs differentially affect common food-borne pathogenic bacteria and lactic acid bacteria. Food Control. 2018;92:437-443.

11. Zhang WD. Systems Biology and its Application in TCM Formulas Research. Academic press; 2018.

12. Hugon P, Lagier JC, Colson P. Repertoire of human gut microbes. Microb Pathogenesis. 2017;106:103-112.

13. Geller LT, Barzilyrokni M, Danino T, et al. Potential role of intratumor bacteria in mediating tumor resistance to the chemotherapeutic drug gemcitabine. Science. 2017;357(6356):1156-1160.

14. Cacho RA, Tang Y, Chooi YH. Next-generation sequencing approach for connecting secondary metabolites to biosynthetic gene clusters in fungi. Front Microbiol. 2015;5:774.
15. LiP, Mao Z, Lou J, eta1. Enhancement of diosgenin production in Dioscorea zingiberensis cell cultures by oligosaccharides from its endophytic fungus Fusarium oxysporum Dzf17. Molecules. 2011;16(12):10631-10644.

16. Richardson SN, Nsiama TK, Walker AK, et a1. Antimicrobial dihydrobenzofurans and xanthenes from a foliar endophyte of Pinus strobus. Phytochemistry. 2015;117:436-443.

17. Tejesvi M, Birgitte A, Nikolinka A. MB 1533 is a defensin-like antimicrobial peptide from the intracellular meristem endophyte of scots pine Methylobacterium extorquens DSM 13060. J Microb Biochem Technol. 2015:8(1):445.

18. Pan F, Su TJ, Cai SM. Fungal endophyte-derived Fritillaria unibracteata var. wabuensis: diversity, antioxidant capacities in vitro and relations to phenolic, flavonoid or saponin compounds. Sci Rep. 2017;(7):1-14. 